\title{
El método del proyecto en los estudios de grado en Comunicación Audiovisual. El Festival de Cortos AdN como ejemplo aglutinador de competencias
}

\author{
Mar Ramos RodRíGuez \\ Universidad Nebrija de Madrid \\ mramos@nebrija.es
}

\section{Resumen:}

En este artículo se reflexiona sobre el aprendizaje mediante proyectos como estrategia de enseñanza en los estudios de grado en Comunicación Audiovisual, a través del recorrido de una actividad, hasta ahora extra-curricular: el Festival de Cortometrajes AdN, en la universidad Nebrija de Madrid. A través de estas líneas se intentará determinar si es una fórmula de éxito para la obtención de las competencias exigidas para un graduado o graduada en Comunicación Audiovisual y de la incorporación del mismo dentro de la malla curricular en los actuales planes de estudio.

Palabras clave: Aprendizaje por proyectos; educación superior; competencias

\section{The method of project in the studies of the degree in Audiovisual Communication}

\begin{abstract}
:
This article reflects on learning through projects such as teaching strategy in the studies of the degree in Audiovisual Communication, through the course of an activity, until now extra-curricular: Festival de Cortometrajes AdN, at the University of Nebrija in Madrid. Through these lines, will attempt to determine whether it is a successful formula for obtaining the skills required for a graduate or graduate in Audiovisual Communication and the incorporation of it within the curriculum in current curricula.
\end{abstract}

Key Words: Learning through projects; higher education; competencies

\section{Referencia normalizada:}

Ramos Rodríguez, M. (2014): El método del proyecto en los estudios de grado en comunicación audiovisual. El festival de cortos AdN como ejemplo aglutinador de competencias. Historia y Comunicación Social. Vol. 19. Núm. Especial Febrero. Págs. 779-790.

Sumario: 1. Introducción. 2. El contexto. 3. La metodología. 4. La experiencia. 4.1. Participación de cortometrajes. 4.2. Participación en la organización. 5. Resultados. 6. Conclusiones. 7. Referencias bibliográficas. 


\section{Introducción}

El nuevo panorama en que la Universidad se halla desde la aparición del Espacio Europeo de Educación Superior (EEES), ha provocado el abandono de metodologías docentes centradas en la mera transmisión de conocimientos clásica y buscar nuevas prácticas educativas, que aunque no tan nuevas, no tenían recorrido en las aulas universitarias. Prácticas que se fundamentan en estrategias basadas en los estudiantes como protagonistas de su propio aprendizaje y que incorporan no solo habilidades para desarrollar un camino que conduzca a la adquisición del conocimiento de manera autónoma, sino también aptitudes para la aplicación práctica del mismo en ambientes de trabajo.

$\mathrm{Y}$ en estas prácticas se encuentra el aprendizaje por proyectos, una estrategia de enseñanza en el que los estudiantes planifican, desarrollan y evalúan proyectos que les permiten aplicar los conocimientos adquiridos en una situación real, que en muchos casos es una experiencia profesional completa. Se convierte, pues en una actividad que sale del aula y se convierte en una estrategia profesional. Para su desarrollo se realizan diferentes actividades interdisciplinares, normalmente realizadas en periodos de tiempo más largos de lo que es habitual en otro tipo de actividades curriculares y en las que los estudiantes son los verdaderos conductores, y los profesores se tornan en asesores. Se busca un papel más activo en ambos y una adquisición de conocimientos y competencias basado en la búsqueda, en la aplicación, en la experimentación, en la resolución de problemas, adoptando además un papel innovador y transformador que convierta a los estudiantes en nuevos creadores ante nuevos escenarios y realidades. (Cuadrado y Fenández Antelo, 2008: 200)

Se trata de mantener a los estudiantes comprometidos y motivados ante proyectos que suponen retos e implicación por parte de los mismos. Proyectos que aúnen materias y conocimientos con aptitudes y habilidades para conseguir un trabajo común que conduzca a un vistoso resultado. Se trata de actividades que son altamente motivadoras para los estudiantes, lo que hace que estén muy involucrados en los mismas, sintiéndolas como algo suyo, y no solo de forma individualista, sino como un proyecto de grupo, en el que se aúnan las fortalezas individuales de los integrantes en aras de un bien común.

Según un trabajo realizado por el Northwest Regional Educational Laboratory durante el año 2003 y que recogieron publicado en el documento "Project-Based Instruction: Creating Excitement for Learning"1, los proyectos auténticos tienen en común los siguientes elementos específicos:

- Centrados en el estudiante y dirigidos por el estudiante.

- Claramente definidos, un inicio, un desarrollo y un final

1 [25-10-2013] http://educationnorthwest.org/webfm_send/460 
- Contenido significativo para los estudiantes; directamente observable en su entorno

- Problemas del mundo real

- Investigación de primera mano

- Sensible a la cultura local y culturalmente apropiado

- Objetivos específicos relacionados con el proyecto educativo y los estándares del currículo

- Un producto tangible que se pueda compartir con la audiencia objetivo

- Conexiones entre lo académico, la vida y las competencias laborales

- Oportunidades de retroalimentación y evaluación por parte de expertos

- Oportunidades para la reflexión y la auto-evaluación por parte del estudiante

- Evaluación o valoración auténtica

Como principales beneficios se encuentran:

- Preparar a los estudiantes para la vida laboral. Adquisición de competencias y habilidades como el trabajo en equipo, la toma de decisiones, la gestión del tiempo,...

- Aumento de la motivación y la participación

- El aprendizaje colaborativo permite compartir ideas, expresar soluciones y opiniones, negociar con diferentes estamentos...

- Aumentar las habilidades sociales y de comunicación

- Aumentar la autoestima. El valor de un proyecto propio fuera del aula.

- Permitir el uso de fortalezas individuales de aprendizaje y de sus diferentes enfoques hacia este.

- Uso de las tecnologías de una forma práctica, para un uso real.

La realización de un buen trabajo elaborado con el método del proyecto, se torna en un resultado aglutinador de competencias tanto generales como específicas, que lo convierte en un magnífico aliado para las prácticas universitarias en las que tienen que confluir un gran número de habilidades y competencias. Dichas prácticas se pueden traducir en el currículo universitario en el trabajo fin de grado, en el que hay que demostrar todas las competencias que contempla el plan de estudios de un graduado o graduada en Comunicación Audiovisual. Y esa es la propuesta que se hace desde estas líneas, con una actividad extra-académica que se realiza en la Universidad Nebrija con los estudiantes de la facultad de Ciencias de la Comunicación, y que se puede resumir como un proyecto pre-profesional realizar paralelamente a las aulas y que es, sin duda, una fórmula de éxito. 


\section{El contexto}

Se hace necesario para entender la propuesta motivo de análisis en estas líneas, contextualizarla dentro del marco en el que se realiza. El Festival de Cortometrajes AdN se enmarca dentro de los estudios de la Facultad de Ciencias de la Comunicación de la Universidad Nebrija, que es una institución universitaria privada de pequeño tamaño. Se define como laica e independiente y cuenta con dos campus, uno en Madrid capital, en el parque de la Dehesa de la Villa y otro en Hoyo de Manzanares, en la Berzosa. En este último campus es donde se encuentra establecida la facultad de Ciencias de la Comunicación. El Festival de Cortometrajes AdN nació en el curso académico 1998-1999. Lo hizo por tanto dentro de los planes de estudio de licenciatura, anteriores a la implantación del grado con la implantación del Espacio Europeo de Educación Superior (EEES). En el momento de su nacimiento, no se pensó en esta actividad como en una actividad estrictamente docente, sino que de forma "altruista" tanto el alumnado como el profesorado, la contemplaron como una oportunidad semi-profesional de poder proyectar en un cine el resultado de un trabajo en equipo, en forma de cortometraje. Estas pequeñas obras audiovisuales eran realizadas y producidas, sobre todo por los alumnos de último curso de la licenciatura, y con la supervisión de algunos profesores que en el aula contribuían al proceso y su posterior seguimiento. Con esta iniciativa se buscaba que pudieran ser visionadas fuera del aula y en un formato que fuera profesional. Esto implicaba, además de una proyección en una sala y con público, ir más allá y tener una valoración que premiara las obras y por consiguiente, un jurado que votara y unos premios que dieran aún más motivación a los estudiantes para presentar sus cortometrajes.

El festival fue creciendo, provocado mayoritariamente por el entusiasmo que provocó tanto en los estudiantes como en los profesores, y lo hizo en todos los sentidos: el visionado comenzó siendo en la sala de Conferencias del Campus de la Berzosa de la Universidad Nebrija, donde se encuentra la facultad de Ciencias de la Comunicación, para pasar después al Teatro Bulevar, en el Centro Cultural de Torrelodones, población próxima al mencionado campus, y un par de años más tarde a los cines Verdi en Madrid; aunque el momento de éxito para el festival llegó en la VII edición, cuando se comenzó a celebrar en el Cine Capitol, en la cinematográfica Gran Vía madrileña. Y el jurado que otorga los premios en el festival se fue haciendo cada vez más experto y ajeno a las aulas, con lo que grandes profesionales (guionistas, productores, actores y actrices, realizadores,...) a lo largo de estos años han colaborado con la universidad en esa labor de forma entregada y apasionada ante las inquietudes de los nuevos creadores audiovisuales. Y como no podía ser menos, el diseño y la elaboración de la entrega de premios también ha crecido, y con ello la implicación y el trabajo de los alumnos: nuevas ideas, guiones, colaboraciones con estudiantes de otros grados.

En el pasado curso 2012-2013 el festival ha celebrado su decimoquinta edición. A partir de ésta, el festival se celebra de forma bianual, pero no es por falta de entusiasmo en los estudiantes, sino por un tema logístico de la facultad: esta actividad convive 
con otra de la disciplina publicitaria, el Festival Jóvenes Tocados por la Publicidad, y la dimensión que han adquirido ambas no permiten hacer las dos anualmente con la exigencia de tiempo y esfuerzo que requieren tanto para el alumnado como para el profesorado que las supervisa.

\section{La metodología}

El análisis propuesto en estas líneas intenta ser un primer paso de reflexión para algo que se está trabajando dentro de la facultad de Ciencias de la Comunicación: la posibilidad de cruzar los Trabajos Fin de Grado que incorpora el plan de estudios del grado en Comunicación Audiovisual con el trabajo realizado por los estudiantes de dicho grado en el Festival de Cortometrajes AdN.

Se trata pues, de analizar un caso de forma intrínseca y aplicarle las características que tiene el método del proyecto y que se han incluido en el primer capítulo, de forma que podamos discernir si los estudiantes adquieren las competencias exigidas en la asignatura Trabajo Fin de Grado, dentro de la malla curricular del plan de estudios del grado en Comunicación Audiovisual. Para ello haremos un estudio de observación para ver si podemos adaptar esta actividad, ahora fuera del sistema de créditos, dentro de los estudios universitarios en los que se realiza.

Este análisis de observación, junto con su adaptación a la metodología del proyecto de una forma comparativa, nos hará obtener una conclusión de si podemos establecer y son útiles esta metodología y la búsqueda de proyectos que sean de interés y motivación para los estudiantes, una buena alternativa dentro del nuevo paradigma en el que buscamos estudiantes activos, implicados en su propio proceso de aprendizaje, con responsabilidades en la toma de decisiones, partícipes de sus propios recursos y con relaciones colaborativas tanto con profesores como con sus propios compañeros. Un espacio en el que el profesor también cambia de papel y pasa de ser protagonista a asumir un papel de colaborador en el proceso de enseñanza.

\section{La experiencia}

Como ya se ha comentado anteriormente, el Festival de Cortometrajes AdN se realiza fuera del plan de estudios del grado en Comunicación Audiovisual, lo que significa que no se corresponde con ninguna asignatura concreta en la malla curricular. Sin embargo, la finalidad que desde sus comienzos se buscó, tanto por parte del claustro docente como del alumnado, fue que se convirtiera en una práctica global de todos los conocimientos adquiridos en licenciatura primero y en los estudios de grado en la actualidad. Esto se conseguía tanto con la producción y realización de los cortometrajes presentados a concurso, como con la organización del festival y se buscaba con todo ello un escenario real en el que visionar los trabajos a concurso y el 
reconocimiento por parte de estudiantes, egresados y profesionales, y por tanto, futuros empleadores, del trabajo realizado por los estudiantes (ya incipientes egresados en muchos casos) de los estudios de nuestra facultad.

Según Gardner, H. ${ }^{2}$

Fuera del marco de la escuela, los individuos aprenden habilidades y cuerpos de conocimiento en contextos ricos, sustentadores y esencialmente "naturales", en los que la información es sumamente redundante y el feedback inmediato y normalmente muy apropiado. En cambio en el interior de la mayoría de los marcos escolares, el acento recae directamente en el aprendizaje de la información separada del contexto en el que es probable que se utilice.

Los dos marcos presentados por Gardner, la universidad (escuela) y el ámbito fuera de ella, se entremezclan en la actividad propuesta enriqueciéndola con lo que cada uno de ellos puede aportar para el aprendizaje y la adquisición de competencias y habilidades. Así, contexto, habilidades, información e individuos forman una red perfectamente ensamblada, que sale al exterior, a los ámbitos y escenarios en los que se desarrollará la práctica profesional de los hoy estudiantes.

El Festival de Cortometrajes AdN se vertebra en dos líneas: la de concursante y por tanto, aspirante a premio, y la de participante en la organización del festival. En ambas, los proyectos tienen en común muchas características de las que hemos determinado para el método del proyecto, y que en estas líneas se intenta demostrar de gran valor como una magnífica herramienta pedagógica en el espacio universitario. Sin embargo, hay una diferencia esencial, que hace que para contemplar ambas como una actividad dentro de la malla curricular, hubiera que determinar de forma más precisa las condiciones de trabajo y de consideración de los proyectos presentados a concurso: el hecho de que actualmente no es necesario que todos los integrantes del equipo de los cortometrajes sean estudiantes de la universidad. Es por ello que en la revisión del plan de estudios de Comunicación Audiovisual propuesto el pasado curso 2013-2014 para verificación por parte de la Agencia Nacional de Evaluación de la Calidad (ANECA), se hayan incluido asignaturas como Taller de Cortometrajes, que sirvan de germen y desarrollo de las obras audiovisuales presentadas a concurso, y que estando diseñadas dentro del aula, se produzcan en grupos de estudiantes con un fin común.

La segunda de las líneas de esta actividad sí está estructurada ya desde su nacimiento como un proyecto paralelo al aula, con profesores mentores que van guiando a los estudiantes por el camino a recorrer, y es la organización del festival.

\subsection{Participación de cortometrajes}

Para la participación en el Festival como cortometrajista, la premisa es cumplir las bases del festival, que son:

2 GARDNER, H. : Educación artística y desarrollo humano. (Pg. 53) 
- Que el director, productor o guionista (pudiendo ser la misma persona) sean estudiantes en la Universidad Nebrija o licenciado/graduado en la misma para la categoría Mejor Cortometraje Antiguo Alumno.

- Que los trabajos presentados no lo hayan sido en anteriores ediciones.

- Que estén subtitulados al castellano si el idioma utilizado fuera distinto a éste.

- Que cumplan los requisitos técnicos y administrativos de entrega.

- Que no excedan los 5 minutos de duración, incluidos los títulos de crédito.

Los cortometrajes pueden optar a las categorías de Mejor Cortometraje AdN, Mejor Dirección, Mejor Guión, Mejor Montaje y Postproducción y Premio del Público. Existen, además, dos categorías especiales: Mejor Cortometraje Antiguo Alumno y Premio de Honor AdN. El primero es un premio que busca no perder relación con nuestros egresados, hoy profesionales y por tanto, posibles empleadores. Seguir teniendo vinculación con nuestros antiguos alumnos es uno de nuestros pilares y le da mucha fuerza a esta actividad. Respecto a los premios de honor, a lo largo de la historia del festival se han dado a profesionales de la talla de Juan Mariné, Gil Parrondo, Luis Tomás Melgar, Antonio Ozores, Pepe Quetglas, Fernando Méndez Leite, Nacho Vigalondo, María Reyes, Alberto Iglesias y Enrique Urbizu. Tener este elenco de personas vinculadas al mundo del cine y la televisión recibiendo un premio de manos de nuestros estudiantes es un hecho que les ilusiona enormemente y hace que sea una motivación muy grande para su participación en el festival.

Habitualmente, los estudiantes organizan sus propios rodajes, procesos de pre-producción y postproducción, bajo su responsabilidad, su criterio y su propia forma de trabajo. Escriben sus guiones, realizan las planificaciones pertinentes y organizan sus rodajes y fases de postproducción. Una vez tienen finalizadas sus obras y se adecúan a las normas que establecen las bases del festival, presentan el resultado a concurso y solo falta esperar los resultados. Algunos de los trabajos están realizados bajo el paraguas de la universidad por utilizar materiales, ayuda de becarios o profesores, y muchas veces poco más. Otros son totalmente externos y el ámbito universitario no tiene ninguna implicación en ellos. Una vez recibidos todos los cortos participantes, se realiza una primera selección en el caso de que el número de los mismos sea muy elevado. La razón es únicamente logística, y atiende al número de minutos que se tienen de proyección en sala para el día de la gala. El fin es visionar el mayor número de cortos ese día, pues este es el primer premio que reciben los participantes, pero desgraciadamente, si el metraje total de los cortos presentados es superior al horario que tenemos de proyección, se hace ineludible una primera selección, que normalmente suele ser de carácter técnico: deficiencias de sonido, incompatibilidad en el sistema,.. Una vez realizada esa primera criba, si es necesaria (y casi siempre lo es), se envían los trabajos a un jurado de profesionales del sector audiovisual de diferentes órdenes, y ellos son los que determinan los premios otorgados a las piezas que se visionarán en la sala el día de celebración de la gala de entrega de premios. Ese día se vota además el premio del Público, que es votado entre los asistentes y que, como en 
todo buen certamen que tiene esta categoría, es un premio muy valorado por nuestros jóvenes cineastas.

Se trata pues, esta modalidad de participación, como hemos venido diciendo, de la más tangencial al aula puesto que los estudiantes no demandan, en la inmensa mayoría de los casos, ninguna tutorización ni ayuda de parte de profesores ni de la entidad universitaria. Estableceremos una valoración como método del proyecto, ya que creemos que su incorporación en cierta medida al aula, puede favorecer la calidad de los cortometrajes y una mejor asunción de los roles en todo el proceso de producción de los mismos. Esto quiere decir, una mayor profesionalidad, que muchas veces no es asumida aunque los resultados sean dignos para el espacio en el que son visionados.

\subsection{Participación en la organización}

La actividad organizativa del festival está dirigida principalmente por los estudiantes de tercero y cuarto curso, ya que son los cursos antesala a su graduación y posterior inserción en el mundo laboral. Ellos son los protagonistas del diseño y desarrollo del festival y ocupan los puestos relevantes dentro del organigrama. A pesar de que los puestos de dirección estén reservados en cierta forma para ellos, todos los estudiantes de la facultad pueden participar en todo el proceso de organización y realización del festival y de todo el trabajo que va generando mes a mes su desarrollo. Además, hay puestos dentro del organigrama de marcado interés para las otras disciplinas de la facultad: Periodismo y Publicidad y Relaciones Públicas, como pueden ser las relaciones con los medios tanto institucionales como externos o labores de patrocinio o community manager. Además, incluimos desde la implantación de los grados, una nueva materia afín a nuestros estudios, que es la de Artes Escénicas, de donde han salido ya algunos de los presentadores de la gala de premios y bastantes de los cortometrajes presentados a concurso.

En el mes de octubre, al comienzo de las clases, se convoca una reunión con todos los profesores y los estudiantes que voluntariamente tengan intención de participar en la edición de ese año. En esta primera reunión el profesor coordinador explica la dinámica de trabajo y alientan a los estudiantes a trabajar y a que definan en qué punto del organigrama quieren formar parte. Esta es una reunión informal, cuyo interés es informar y motivar respecto a las expectativas que tiene la realización de la nueva edición del festival. Podemos establecer, por la participación obtenida, sobre todo en las dos últimas ediciones, que corresponden a los años en los que se ha implantado el grado, que aproximadamente un 50\% de los estudiantes entre tercer y cuarto curso participan en esta primera reunión. Es una participación muy alta, teniendo en cuenta que un $25 \%$ de nuestros estudiantes de cuarto curso y algunos de tercero están realizando prácticas laborales en empresas, lo que también forma parte de su plan de estudios, y que, por tanto no pueden implicarse de forma efectiva en la organización. 
El organigrama básico para la organización (Imagen 1) es el siguiente:

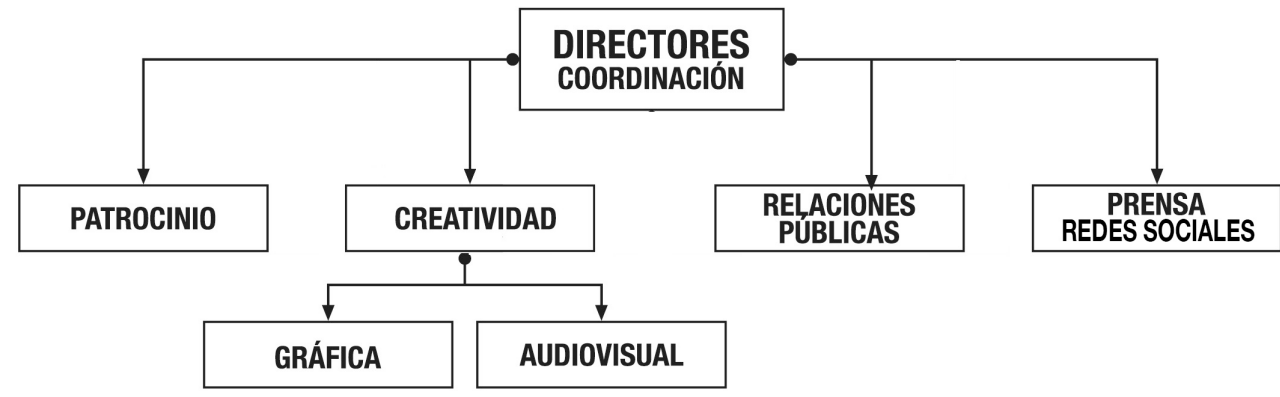

Imagen 1. Fuente: Elaboración propia

Los estudiantes se apuntan al departamento que más les interesa en el plazo de una semana aproximadamente y posteriormente, con una lista cerrada de interesados, se eligen a los responsables de las diferentes áreas, tal como queda reflejado en el cuadro de la Imagen 1. Los alumnos participantes cuentan siempre en este proyecto con la ayuda y la supervisión de los profesores de la facultad. En algunas ediciones, el trabajo de algún departamento ha sido llevado expresamente al aula, como ocurre en los departamentos de Creatividad Gráfica y Audiovisual, donde las ideas son germinadas y elaboradas en asignaturas como Creatividad Publicitaria y Dirección de Arte o Edición y Montaje y Postproducción.

Una vez organizados los departamentos, los responsables se reúnen periódicamente con un profesor responsable, que hará de guía en su trabajo y se convertirá en el nexo con la universidad, que en esta actividad, se convierte en un potencial cliente, lo que da un carácter muy profesional al evento. No debemos olvidar que esta actividad representa un coste en los presupuestos de la facultad y además representa una imagen de la misma, por lo que la actividad ha de ajustarse al dinero asignado y a la supervisión de los órganos rectores. Esto es una práctica muy valiosa, pues en un ambiente más o menos conocido los estudiantes se enfrentan a un cliente real con los problemas que éstos pueden plantear en cualquier desafío laboral que deban afrontar. Las reuniones periódicas producen unos informes de seguimiento y planificación de los departamentos con los directores a la cabeza. Este doble feedback entre los propios estudiantes y los profesores responsables y la universidad como cliente final produce un engranaje muy interesante en cuanto a la resolución de problemas y defensa de intereses se refiere. Además, todo el profesorado implicado mantiene sus propias reuniones con los responsables de la facultad para reportar sobre el desarrollo del festival.

A continuación se describe brevemente la labor realizada por los diferentes departamentos:

- Directores coordinadores. Elegidos por su liderazgo, capacidad de trabajo y responsabilidad entre sus compañeros, serán los encargados de coordinar todos los departamentos y servir de puente con los profesores responsables. 
Son los máximos responsables y también son los encargados de cerrar el acto de entrega de premios con un discurso final el día de la gala.

- Departamento de patrocinio. Tiene que intentar conseguir financiación para el proyecto y controlar el presupuesto asignado. Para ello elaboran un dossier de patrocinio que mueven entre empresas afines a los valores que transmite el festival, como son juventud, espacios de ocio, cine, cultura,... buscando financiación o aportaciones como regalos, patrocinio de alguno de los premios,...

- Departamento de creatividad. Se encargan de diseñar la imagen gráfica del festival con todas las piezas que ellos incluye: carteles, invitaciones, etc. Así como todas las piezas audiovisuales que se proyectarán el día del evento y darán unidad al festival: cabeceras, making of del festival, etc.

- Departamento de Relaciones Públicas. Este departamento se encarga de la organización del día del festival, del protocolo, de la recepción de invitados, la coordinación de azafatas, etc.

- Departamento de Prensa y Redes Sociales. Este departamento funciona como un gabinete de prensa que trabaja bajo la supervisión directa del departamento de Comunicación de la universidad. Se encargan de dar notoriedad a la entrega del Premio de Honor y desde hace un par de ediciones está siendo muy importante la labor realizada por los estudiantes encargados de este departamento a través de las redes sociales.

\section{Resultados}

Una vez descrita la actividad en sus dos vertientes y el éxito que supone entre los estudiantes, que hasta ahora participan simplemente por el valor que tiene para ellos la identidad del festival y lo que supone como orgullo para ellos el haber formado parte de una edición exitosa del mismo, vamos a verificar si las premisas que ha de cumplir esta actividad para estar enmarcada en el método del proyecto se cumplen y si es posible, por tanto, contemplar su desarrollo en un futuro integrado en los planes de estudio del grado en Comunicación Audiovisual de la Universidad Nebrija, dentro del denominado Trabajo Fin de Grado.

\begin{tabular}{|c|c|c|}
\hline \multicolumn{3}{|c|}{ CARACTERÍSTICAS } \\
\hline MÉTODO PROYECTO & $\begin{array}{c}\text { PARTICIPACIÓN DE } \\
\text { CORTOMETRAJES }\end{array}$ & $\begin{array}{c}\text { ORGANIZACIÓN } \\
\text { DEL EVENTO }\end{array}$ \\
\hline $\begin{array}{c}\text { Centrados en el estudiante y dirigidos por } \\
\text { el estudiante. }\end{array}$ & & \\
\hline $\begin{array}{c}\text { Claramente definidos, un inicio, un } \\
\text { desarrollo y un final }\end{array}$ & & \\
\hline $\begin{array}{c}\text { Contenido significativo para los } \\
\text { estudiantes; directamente observable en su } \\
\text { entorno }\end{array}$ & & \\
\hline Problemas del mundo real & & \\
\hline
\end{tabular}




\begin{tabular}{|c|l|l|}
\hline Investigación de primera mano & & \\
\hline $\begin{array}{c}\text { Sensible a la cultura local y culturalmente } \\
\text { apropiado }\end{array}$ & & \\
\hline $\begin{array}{c}\text { Objetivos específicos relacionados con el } \\
\text { proyecto educativo y los estándares del } \\
\text { currículo }\end{array}$ & & \\
\hline $\begin{array}{c}\text { Un producto tangible que se pueda } \\
\text { compartir con la audiencia objetivo }\end{array}$ & & \\
\hline $\begin{array}{c}\text { Conexiones entre lo académico, la vida y } \\
\text { las competencias laborales }\end{array}$ & & \\
\hline $\begin{array}{c}\text { Oportunidades de retroalimentación y } \\
\text { evaluación por parte de expertos }\end{array}$ & & \\
\hline $\begin{array}{c}\text { Oportunidades para la reflexión y la auto- } \\
\text { evaluación por parte del estudiante }\end{array}$ & & \\
\hline Evaluación o valoración auténtica & & \\
\hline
\end{tabular}

Imagen 2. Fuente: Elaboración propia

Según puede verse en el cuadro anterior (Imagen 2), el Festival de Cortometrajes AdN, cumple según el documento "Project-Based Instruction: Creating Excitement for Learning", que hemos tomado como modelo para nuestro análisis desde el comienzo de este artículo, todas las características para ser denominado como un proyecto auténtico. Y lo hace en sus dos modalidades de trabajo, tal y como las hemos desarrollado en el punto anterior.

\begin{tabular}{|c|c|c|}
\hline \multicolumn{2}{|c|}{ BENEFICIOS } \\
\hline MÉTODO PROYECTO & $\begin{array}{c}\text { PARTICIPACIÓN } \\
\text { DE } \\
\text { CORTOMETRAJES }\end{array}$ & $\begin{array}{c}\text { ORGANIZACIÓN } \\
\text { DEL EVENTO }\end{array}$ \\
\hline $\begin{array}{c}\text { Preparar a los estudiantes para la vida } \\
\text { laboral. Adquisición de competencias y } \\
\text { habilidades como el trabajo en equipo, la } \\
\text { toma de decisiones, la gestión del tiempo,... }\end{array}$ & & \\
\hline Aumento de la motivación y la participación & & \\
\hline $\begin{array}{c}\text { El aprendizaje colaborativo permite } \\
\text { compartir ideas, expresar soluciones } \\
\text { y opiniones, negociar con diferentes } \\
\text { estamentos... }\end{array}$ & & \\
\hline $\begin{array}{c}\text { Aumentar las habilidades sociales y de } \\
\text { comunicación }\end{array}$ & & \\
\hline $\begin{array}{c}\text { Aumentar la autoestima. El valor de un } \\
\text { proyecto propio fuera del aula. }\end{array}$ & & \\
\hline $\begin{array}{c}\text { Permitir el uso de fortalezas individuales } \\
\text { de aprendizaje y de sus diferentes enfoques } \\
\text { hacia este. }\end{array}$ & & \\
\hline $\begin{array}{c}\text { Uso de las tecnologías de una forma práctica, } \\
\text { para un uso real. }\end{array}$ & & \\
\hline
\end{tabular}

Imagen 3. Fuente: Elaboración propia 
Por otra parte, los beneficios que aporta un buen proyecto dentro de la metodología docente que persigue la adquisición de competencias y habilidades dentro del currículo de la educación superior, creemos que se cumplen en su totalidad en ambas formas de participación en el festival (Imagen 3).

A modo de resumen de resultados, podemos establecer que el Festival de Cortometrajes AdN es un proyecto auténtico, nacido sin pretensión de ser una actividad articulada dentro del currículo académico, pero que se ha erigido en una metodología docente muy apropiada para la adquisición de competencias dentro de los planes de estudio de la facultad de Ciencias de la Información.

\section{Conclusiones}

El festival de Cortometrajes AdN es una actividad que puede incluirse dentro de la malla curricular del grado en Comunicación Audiovisual y ofrecer Trabajos Fin de Grado que exigen la adquisición de competencias, tanto específicas como generales que se adquieren en todas las materias, de gran calidad:

- $\quad$ Es una actividad que ofrece dos proyectos auténticos que cumplen todas las características propias de esta metodología: la participación en el festival con un cortometraje y la propia organización del festival como evento, que pueden producir productos audiovisuales con sus correspondientes memorias que se convertirían en Trabajos Fin de Grado rigurosos y muy pegados al mundo profesional, como requiere un graduado o graduada en Comunicación Audiovisual.

- El ámbito de compromiso y motivación por parte de los estudiantes lo convierte en una opción muy apropiada para la adquisición de competencias y habilidades.

- $\quad$ Es un proyecto pre-profesional completo, con una auténtica realidad que desarrollar, defender y exhibir.

- Exige investigación y ofrece oportunidades de tener un escenario de exposición en el que mostrar el trabajo de los estudiantes al mundo profesional y al público.

- Estimula el trabajo en equipo en aras de un bien común, con lo que implica una buena gestión del tiempo, de las relaciones humanas y aprender a gestionar la toma de decisiones y la resolución de problemas. 


\section{Bibliografía}

COLÁS BRAVO, P. Y DE PABLOS PONS, J. (Coord.) (2005): La universidad en la Unión Europea. El EEES y su impacto en la docencia. Ed. Aljibe

CUADRADO, Isabel y FERNÁNDEZ ANTELO, Inmaculada (2008): "Nuevas competencias del profesor en el EEES: una experiencia de innovación docente". Revista Electrónica Teoría de la Educación. Educación y Cultura en la Sociedad de la Información (Vol. 9, $\mathrm{n}^{\circ}$ 1). Universidad de Salamanca.

DE MIGUEL DÍAZ, M. (Dir.) (2006): Modalidades de enseñanza centradas en el desarrollo de competencias. Orientaciones para promover el cambio metodológico en el marco del EEES." Ed. Universidad de Oviedo

GARDNER, Howard (1999): Educación artística y desarrollo humano. Ed. Paidós. Ecuador.

LORENTE, J., DOBLAS, P. y ZALDUMBIDE, I.: La competencia en comunicación en el aprendizaje orientado a proyectos (PBL). Actas de Univest 2011. Girona. Disponible en: http://dugi-doc.udg.edu/bitstream/handle/10256/3716/296.pdf?sequence=1 [20-09-2013]

NORTHWEST REGIONAL EDUCATIONAL LABORATORY (2002) "Project-Based Instruction: Creating Excitement for Learning". Consultado en http://www. eduteka.org/AprendizajePorProyectos.php [25-10-2013]

SIERRA SÁNCHEZ, J. Y SOTELO GONZÁLEZ, J. (Coord) (2010): Métodos de innovación docente aplicados a los estudios de Ciencias de la Comunicación. Ed Fragua. Madrid.

ZABALZA, M.A. (2005): Competencias docentes. Conferencia pronunciada en la Pontificia Universidad Javeriana de Cali. Disponible en: http://portales.puj.edu. co/didactica/Archivos/Competencias\%20docentes.pdf. Última consulta [29-092013]

ZABALZA, M.A. (2007): El trabajo por competencias en la enseñanza universitaria. Conferencia UAB. [28-09-2013] 